Background Poor occupational health and safety damages many lives and livelihoods which impedes economic growth and cause extreme poverty. The significance of occupational health and safety is particularly strong in countries like Bangladesh where it is not adequately addressed or explored.

Methods This qualitative study draws data from 15 Life History interviews with workplace exposed disabled, 10 in-depth interviews with high risk environment workers; and key informant interviews with five senior management officials of risky workplaces.

Results The research found that the poorest people tend to take risky work. Availability of cheap unskilled and semi-skilled labour also contributes to encourage employer to employ without complying with safety and health standards. By accepting risky employment, workers are exposure to gradual or sudden impairment of functions, which in many cases limit their future opportunities making them disabled or left them to die because of the increased risk of illness, injury and/or disability. The cost of dealing with the illness in the absence of insurance forces households to spend its resources on medical care depleting its assets and incurring debts. This further led to exclusion, loss of income, dragged further into poverty and eventually to extreme poverty which also transmit intergenerationally. Bribery and illegal practices helped employers to escape such compliances. On the other hand the lack of willingness of the employers to provide an environment in compliance with standards also seem to be a misunderstanding of the benefits of having a safe working environment. Sub-contracting was found to be a potentially harmful practice of the business/industry owners which makes workers more vulnerable.

Conclusions Relations between occupational injury and impairment and how this leads the households into extreme poverty are both interesting and understudied issue in Bangladesh. Further research studies and strong reporting mechanism is also instrumental to cover the paucity in evidence required for the changes in policies and practice. The paper concludes that occupational health and safety in Bangladesh should be a higher priority in discussions of economic growth and extreme poverty.

\section{AN OVERLOOKED PRIORITY: THE OCCUPATIONAL HEALTH AND SAFETY OF MIGRANT WORKERS}

Courtland Robinson, Abdulgafoor Bachani, Casey Branchini Risko. The Johns Hopkins Bloomberg School of Public Health

\subsection{6/injuryprev-2016-042156.385}

Background Over the last decade, the number of international migrants has increased by more than 60\% globally (150 to 232 million). Generally low-skilled or un-skilled, they are concentrated in "3D" (dirty, dangerous, difficult) jobs in the unregulated sector where they face increased risk of illness, injury and abuse. As international migration rates rise, so does the need for the generation of high-quality data. To date, research has centred predominately on the regulated sector, posing a barrier to the development of effective services for this large, yet overlooked population.

Methods This mixed-methods study (Oct. 2013-Jul. 2015) sought to increase the evidence-base on the occupational health and safety of migrants in Kuala Lumpur, Malaysia. More than 2,500 case files documenting labour rights violations were analysed to identify trends and patterns from 2005 to 2015. Indepth interviews $(n=58)$ and focus groups $(n=6)$ were conducted with migrants in the manufacturing, service, construction and domestic work sectors to develop a more nuanced understanding of their experiences with occupational injury and contextual factors impacting care. Interviews covered a range of topics were conducted in a structured, but open-ended manner. Results Findings illustrate that the majority of workers migrated from Bangladesh (43.7\%), Indonesia (12.8\%) and Nepal (7.5\%). $91.3 \%$ experienced working conditions different from what had been promised and only $18.7 \%$ had a regular day off. $88.8 \%$ did not possess their passports and $4.7 \%$ could gain access to them if necessary. Females were more likely to experience verbal $(11.5 \%$ vs. $65 \%)$ and physical (29.8\% vs. $17.6 \%)$ abuse.

Conclusions Findings illustrate the urgent need to build the evidence base on the occupational health and safety risks faced by migrant workers. This information is critical to improving the health of migrant workers globally.

\section{INJURIES AMONG YOUNG MALE MIGRANTS IN THE UNITED ARAB EMIRATES: QUANTIFYING MORTALITY DIFFERENTIALS}

${ }^{1}$ Katharine A Allen, 'W Courtland Robinson, ${ }^{2}$ EIDaw Soliman, 'David Bishai, 'Adnan A Hyder. ' Johns Hopkins School of Public Health, USA; ${ }^{2}$ Dubai Health Authority, UAE

\subsection{6/injuryprev-2016-042156.386}

Background The United Arab Emirates (UAE) is a high-income developing country where an estimated $80 \%$ of the population is expatriates. The vast majority are males between 15 and 45 years of age from South East Asia who migrate for employment. As temporary labourers in the UAE, many find employment in construction and oil where they are exposed to occupational hazards. Little has been documented about injury-associated mortality among this special population, especially in comparison to UAE citizens.

Methods World Health Organisation and Global Burden of Disease Study protocols were followed to conduct a national burden of disease analysis. Mortality data from the Ministry of Health was used to calculate cause-specific injury death rates for males aged 15-45 years. Years of Life Lost (YLLs) were also calculated and analysed by age-group and nationality.

Results Using 2010 data, the overall injury-related mortality rate for migrant males aged $15-45$ years was 28.0 per 100,000 and the leading causes of injury deaths were road traffic injuries $(22.6 \%$ of all deaths), suicides (7.5\%), other adverse effects (5.8\%) and falls (4.3\%). Leading causes of injury YLLs for this group were road traffic injuries $(28,307$ YLLs), suicides $(10,276)$ and other adverse events $(6,186)$. For similarly-aged male UAE citizens, the overall injury-related mortality rate was 65.0 per 100,000 , with the leading causes being road traffic injuries (43.2\% of all deaths), other adverse effects (6\%) and drowning (1.3\%).

Conclusions Road traffic injuries (RTIs) are a major cause of premature mortality among young male residents regardless of nationality. Much of the difference in injury deaths between migrants and citizens is caused by RTIs. However, migrants experience a much greater burden of non-RTI injuries, including falls and intentional injuries like suicides. This study highlights the need for suicide-prevention and occupational-safety programs for young male migrants in the UAE. 